\title{
DO LENSING STATISTICS RULE OUT A COSMOLOGICAL CONSTANT?
}

\author{
M. CHIBA \\ National Astronomical Observatory, JAPAN \\ AND \\ Y. YOSHII \\ Institute of Astronomy, University of Tokyo, JAPAN
}

We present new calculations of the gravitational lensing statistics following recent revised knowledge of the luminosity function and internal velocity dispersion of $\mathrm{E} / \mathrm{S} 0$ galaxies which work as effective lenses for background high-redshift QSOs. We show that the theoretical prediction of the lensing statistics is much smaller than previously expected. In sharp contrast with the earlier statistics supporting an $\Omega_{0}=1$ universe, the reported small lensing probability from the Hubble Space Telescope (HST) snapshot lens survey is in best agreement with a low-density, flat universe with $\Omega_{0} \simeq 0.2$ and $\Omega_{0}+\lambda_{0}=1$. The age of this universe, combined with the $H S T$ measurement of a high value of the Hubble constant $H_{0}$, can be reconciled with the age of the oldest globular clusters in the Milky Way (ApJ, 1997, Vol. 489, in press).

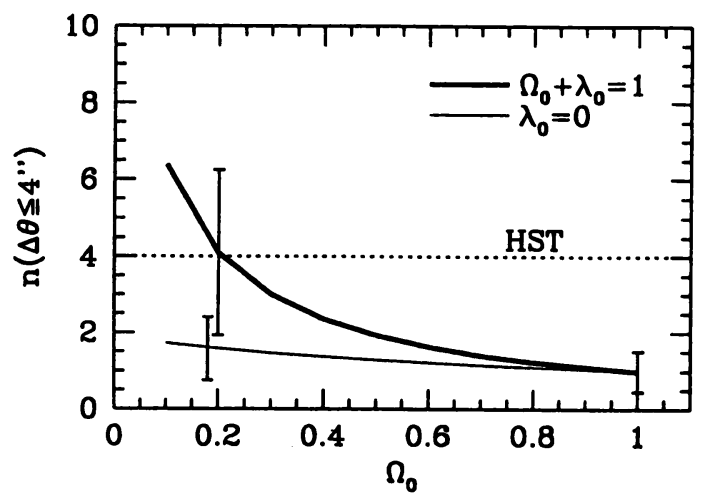

Figure 1. The number of lensed QSOs with $\Delta \theta \leq 4^{\prime \prime}$ as a function of $\Omega_{0}$ for a universe with $\lambda_{0}=0$ (thin line) and a flat universe with $\Omega_{0}+\lambda_{0}=1$ (thick line). 